Mathematical and Computational Applications, Vol. 16, No. 1, pp. 22-30, 2011.

(C) Association for Scientific Research

\title{
A MINERAL CLASSIFICATION SYSTEM WITH MULTIPLE ARTIFICIAL NEURAL NETWORK USING K-FOLD CROSS VALIDATION
}

\author{
Nurdan Akhan Baykan ${ }^{1}$, Nihat Y1lmaz ${ }^{2}$ \\ ${ }^{1}$ Selçuk University, Department of Computer Engineering, Konya, Turkey \\ ${ }^{2}$ Selçuk University, Department of Electric-Electronics Engineering, Konya, Turkey \\ nurdan@selcuk.edu.tr,nyilmaz@selcuk.edu.tr
}

\begin{abstract}
The aim of this study is to show the artificial neural network (ANN) on classification of mineral based on color values of pixels. Twenty two images were taken from the thin sections using a digital camera mounted on the microscope and transmitted to a computer. Images, under both plane-polarized and cross-polarized light, contain maximum intensity. To select training and test data, 5-fold-cross validation method was involved and multi layer perceptron neural network (MLPNN) with one hidden layer was employed for classification. The classification of mineral using ANN proved \%93.86 accuracy for 400 data. In second study, for each of the 5 different mineral considered, 5 different network models were implemented. Size of data set was same with previous data. Each network model was differed from each other. Also 5-fold-cross validation method was involved to select data and MLPNN with one hidden layer was used. The classification accuracy of mineral using different ANN is $\% 90.67 ; \% 96.16 ; \% 93.91 ; \% 92 ; \% 97.62$ for quartz, muscovite, biotite, chlorite and opaque respectively.
\end{abstract}

Key Words - Thin section, Mineral, Microscope, Artificial Neural Network, Cross Validation

\section{INTRODUCTION}

Identification and classification of minerals are the fundamental of the mining and processing of minerals. They are important for the naming rocks. Traditionally, these works are made by the mineralogist using an optical microscope. Color and birefringence properties under polarized light are the basic information for the identification for the naming rocks [1], [2].

Image processing is a technology having varieties of application in the all research fields [3], [4], [5]. But they are at present limited in the mineral processing. In this study, minerals were identified by using microscopic thin section images and classified using color parameters.

Artificial neural networks (ANNs) have been used in a great number of applications in recent years [6], [7], [8], [9]. The application of ANNs has opened new area for solving problems not resolvable by other techniques [10]. A feed forward multi layer perceptron neural network (MLPNN) is the most popular model and the backpropagation algorithm is a widely used to train the MLPNN. A feed-forward MLPNN 
trained with back-propagation algorithm is suitable for classification and therefore was chosen for this study.

Using ANN in the mineral industry is becoming limited. This study shows promising results on the classification of minerals using color parameters. For this aim, a computer controlled microscopy system is designed and provided data from the system are analyzed. The accuracy of developed recognition system by using ANN was between 90.67 and $97.62 \%$.

In the following sections, data acquisition methods and the structure of the neural network for mineral identification using multi layer perceptron will be described. The experimental results and the conclusion will then be presented.

\section{MATERIALS and METHOD}

\subsection{Data Acquisition}

In this study, a digital camera and a conventional microscope with rotating experimental stage was used [11]. The rotating experimental stage is rotated from 0 to 360 degrees by 1 degree increments, while polarizer and analyzer remain crossed to each other in a vertical direction during the analysis. Images were captured as the experimental stage rotated. Basic flow diagram is shown in Figure 1.

In this work, first our image data were gathered using a digital camera mounted on the microscope and then they transmitted to a computer.

Images were taken both plane-polarized and cross-polarized light and contain the maximum intensity values. For finding the maximum intensity image, at every 1 degree increment image was taken and then compared with the previous images. For analysis, first an expert checks the images, decided the area of thin section to be studied and identified which one is which mineral. All images have been stored 450x370 pixel dimension with 150 dpi resolution in RGB format. James Swift microscope provided with a polarizer and a modified analyzer was used for observation of thin sections. A Videolab 3 MP cameras specialized for microscopes mounted on the microscope was used for image data collection and images were transmitted to a computer by Inca software.

In our study, twenty-two digital images were taken from nine thin sections. Thin sections were taken from igneous and metamorphic rocks and a total of 5 common minerals were used namely, quartz, muscovite, biotite, chlorite, and opaque. Quartz was found in all images. Muscovite, biotite, chlorite and opaque was found in twenty, four, twelve and seven images, respectively. First, a median filter was applied to images for noise reduction and then histogram was equalized. 


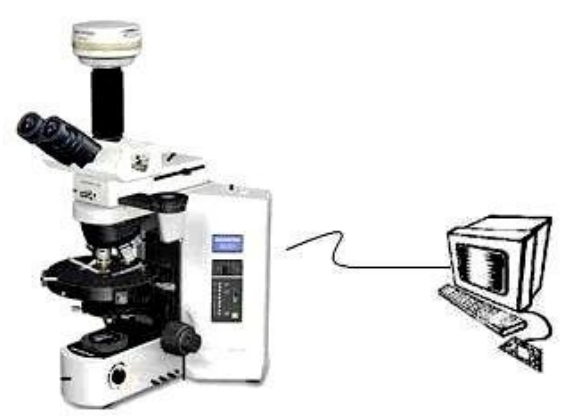

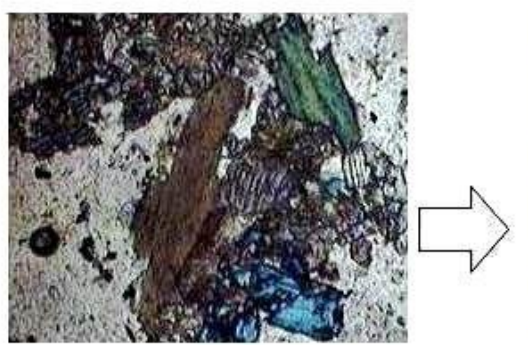

Crossed-polarized image

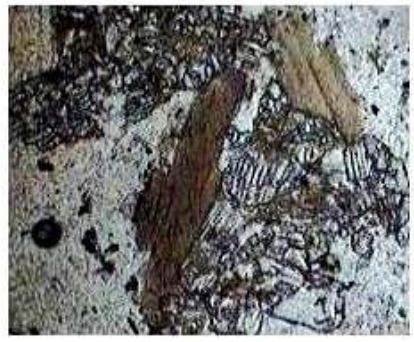

Plane-polarized image

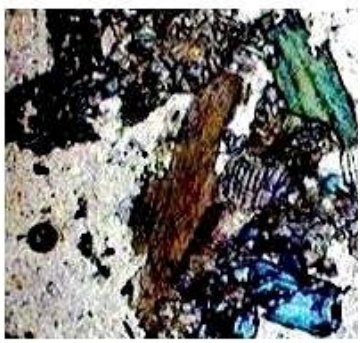

Crossed -polarized image with mes

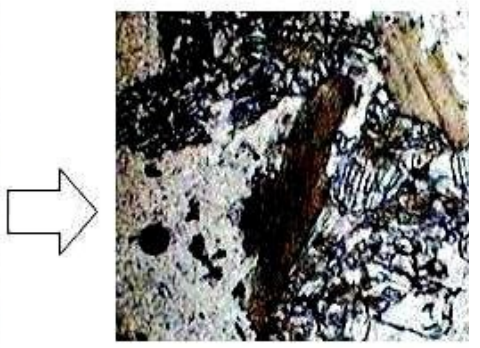

Plane-polarized image with mediat

\begin{tabular}{|l|c|c|c|c|c|c|}
\hline \multirow{3}{*}{ Mineral } & \multicolumn{6}{|c|}{ Pixel Values } \\
\cline { 2 - 7 } & \multicolumn{2}{|c|}{$\mathbf{R}$} & \multicolumn{2}{c|}{ G } & \multicolumn{2}{c|}{ B } \\
\cline { 2 - 7 } & CP Im & PP Im & CP Im & PP Im & CP Im & PP Im \\
\hline Quartz & 219 & 219 & 223 & 198 & 206 & 202 \\
\hline Quartz & 198 & 215 & 215 & 170 & 190 & 182 \\
\hline$\ldots \ldots .$. & $\ldots$ & $\ldots$ & $\ldots$ & $\ldots$ & $\ldots$ & $\ldots$ \\
\hline Muscovite & 89 & 12 & 36 & 154 & 146 & 101 \\
\hline Muscovite & 235 & 146 & 134 & 215 & 239 & 206 \\
\hline$\ldots \ldots \ldots$ & $\ldots$ & $\ldots$ & $\ldots$ & $\ldots$ & $\ldots$ & $\ldots$ \\
\hline Biotite & 101 & 61 & 36 & 57 & 28 & 36 \\
\hline Biotite & 33 & 57 & 49 & 40 & 20 & 28 \\
\hline$\ldots \ldots .$. & $\ldots$ & $\ldots$ & $\ldots$ & $\ldots$ & $\ldots$ & $\ldots$ \\
\hline Chlorite & 65 & 134 & 53 & 20 & 32 & 32 \\
\hline Chlorite & 85 & 154 & 73 & 32 & 53 & 53 \\
\hline$\ldots \ldots . .$. & $\ldots$ & $\ldots$ & $\ldots$ & $\ldots$ & $\ldots$ & $\ldots$ \\
\hline Opaque & 8 & 8 & 16 & 8 & 8 & 16 \\
\hline Opaque & 4 & 8 & 20 & 4 & 4 & 6 \\
\hline$\ldots \ldots \ldots$ & $\ldots$ & $\ldots$ & $\ldots$ & $\ldots$ & $\ldots$ & $\ldots$ \\
\hline
\end{tabular}

CP. Im: Crossed-po PP Im: Plane-polar

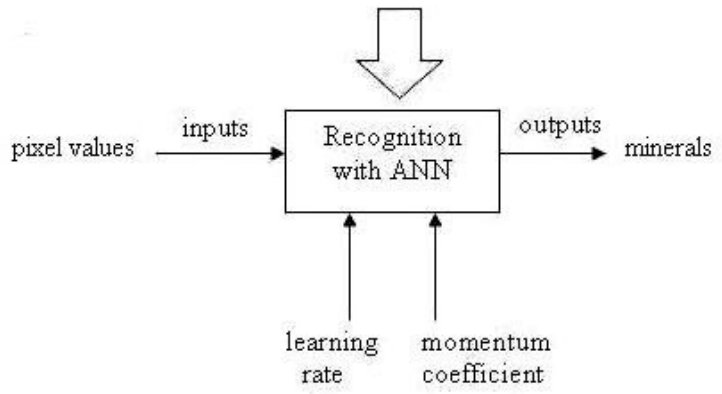

Figure 1. Basic diagram of study

All images were captured using Red, Green and Blue (RGB) components. In the RGB model, each color appears, in its primary spectral components of red, green and blue. This model is based on a Cartesian coordinate system. It can be represented using a normalized cube, where the black is at the origin point $(0,0,0)$ and white is at the 
corner farthest from the origin $(1,1,1)$. The color subspace of interest is the cube shown in Figure 2 [12], in which RGB values are at three corners; cyan, magenta and yellow are at three other corners. For convenience, the assumption is that all color values have been normalized so that the cube shown in Figure 2 is the unit cube. That is, all values of $\mathrm{R}, \mathrm{G}$ and $\mathrm{B}$ are assumed to be in the range $[0,1][12],[13]$.

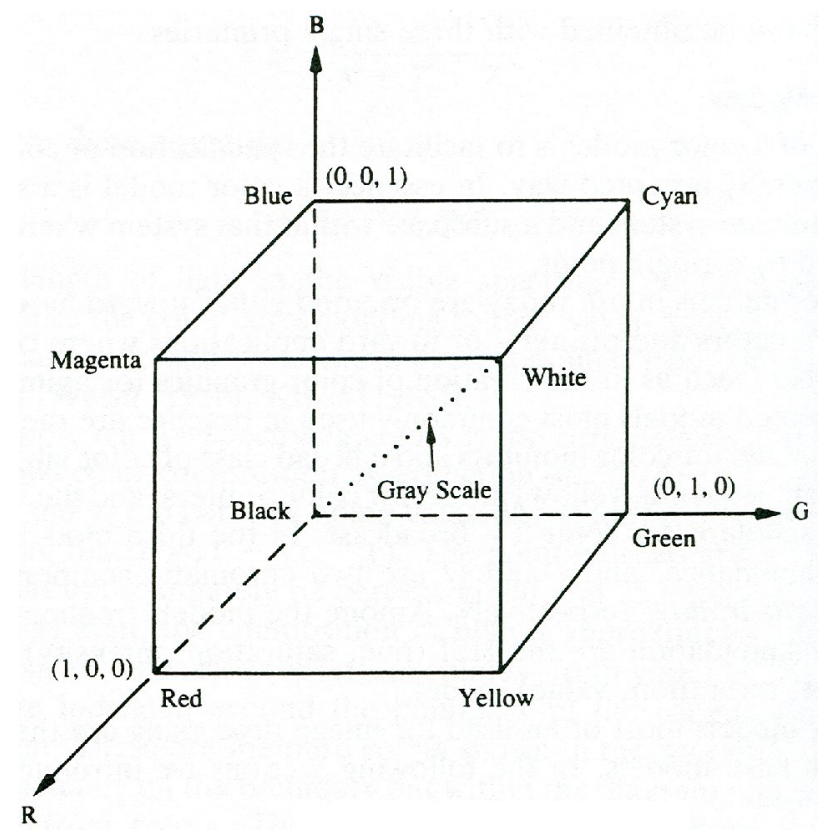

Figure 2. RGB color cube

\subsection{Artificial Neural Network}

Artificial neural networks (ANNs) are biologically inspired intelligent techniques. They have generally a number of simple and highly interconnected neurons organized into layers [14]. ANNs can be trained to recognize patterns and the nonlinear models developed during training allow neural networks to generalize their conclusions and to make application to patterns not previously encountered [15], [16]. Feed forward neural networks are the basic type of neural networks and the multi layer perceptron neural network (MLPNN) is used most [17]. As shown in Figure 3, a MLPNN consists of a layer of input neurons, a layer of output neurons and one or more hidden layers. Layers can have different number of neurons. 


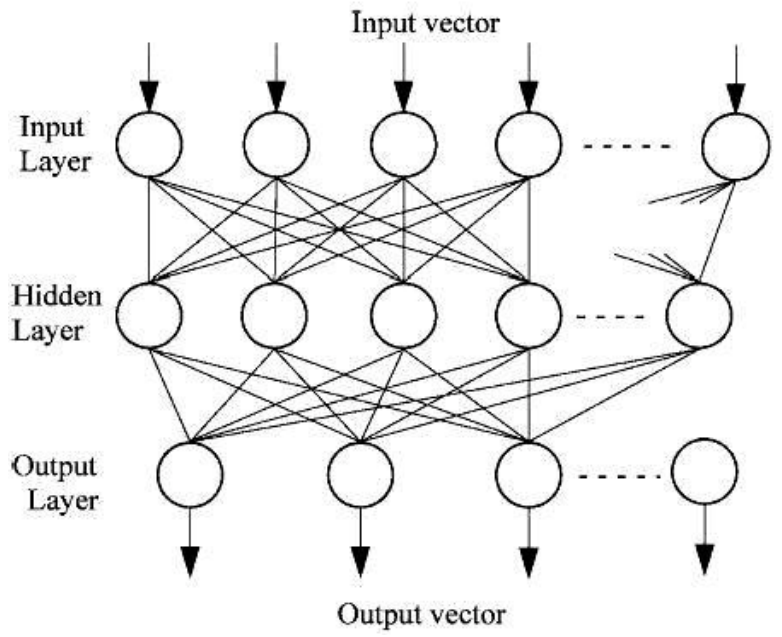

Figure 3. Structure of MLPNN

In MLPNN, each neuron $j$ in the hidden layer sums its input signals $x_{i}$ after multiplying them by the strengths of the respective connection weights $w_{i j}$ and computes its input $I_{j}$ as a function of the sum described as

$$
I_{j}=\sum w_{j i} x_{i}
$$

For its output $y_{j}$, an activation function is used to $I_{j}$ and computes its output as

$$
y_{j}=f\left(\sum w_{j i} x_{i}\right)=f\left(I_{j}\right)
$$

where, $f$ is activation function that is necessary to transform the weighted sum of all signals impinging onto a neuron. The activation function $(f)$ can be a simple threshold function, or a sigmoid, hyperbolic tangent, or radial basis function [8], [18]. But derivative function is preferred because in back propagated process, a derivation of the function is used.

For training MLPNN, back propagation (BP) algorithm is used. In BP algorithm, error calculated as the sum of squared differences between the desired value and the actual value of the output neurons is propagated through the layers of neurons to update the weights [19]. The error of all output neurons $E$ is

$$
E=\frac{1}{2} \sum_{j}\left(y_{d j}-y_{j}\right)^{2}
$$

where $y_{d j}$ is the desired value of output neuron $j$ and $y_{j}$ is the actual output of that neuron. Each weight is adjusted to reduce $E$ as rapidly as possible [8]. 
In this study, feed forward MLPNN with gradient descent with momentum and adaptive learning rule back propagation training algorithm was used. In the hidden layer the activation function $f$ was the tangent sigmoid and in the output layer $f$ was the logarithmic sigmoid function. The initial values of momentum coefficient and the learning rate were taken as 0.8 and 0.1 , respectively. ANN was implemented by using MATLAB version 7.0.4 with neural networks toolbox. Input parameters were scaled to a range of $[-1,1]$ and the output values for each mineral were presented as probabilities in the range of $[0,1]$. Networks were trained from 1000 epoch to 10000 epochs by step 1000 epoch. The training of the network was done using color parameters of example grains and their corresponding mineral type. Three color parameters were extracted from same thin section images which are taken both under crossed-polarized and planepolarized light. A total of 6 input parameters were available for the ANN. To find a computationally less-expensive solution, several networks were constructed. The best results were obtained from a network which was designed as 3 layers with 6 neurons as input parameters for RGB as shown in Figure 4.

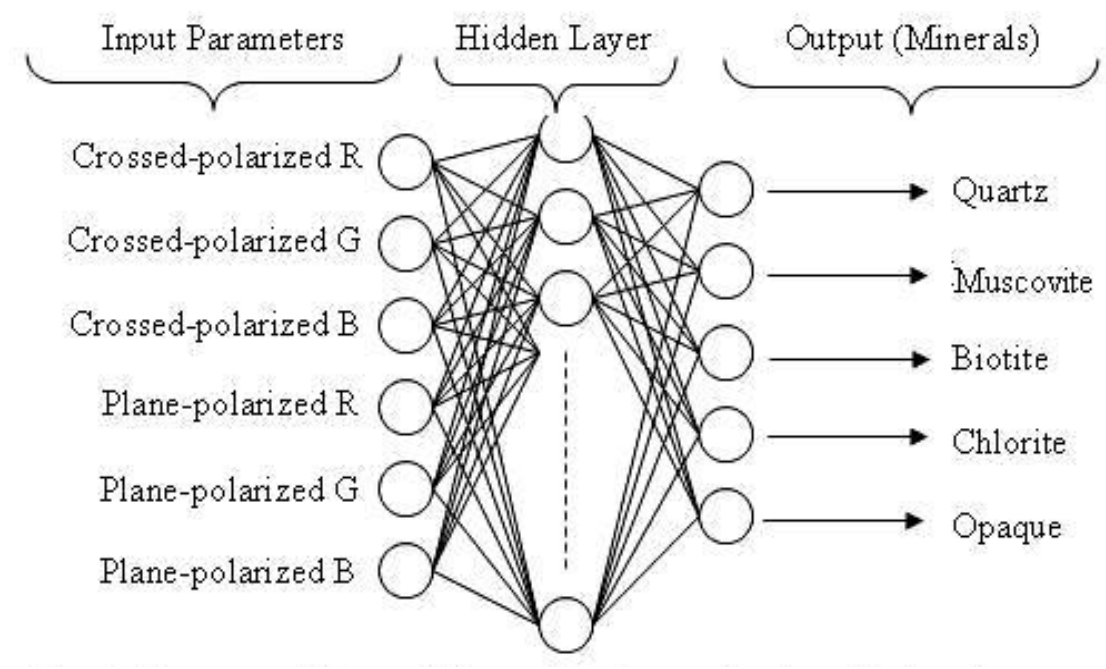

Figure 4. Representation of used neural network structure

\subsection{Cross Validation}

In the training of neural network, $k$-fold cross validation is used to make the test result more meaningful and reliable [20]. In $k$-fold cross-validation, the whole original data is randomly partitioned into $k$ equal size sub samples. Of the $k$ sub samples, in each case, each of the $k$ sub samples is used as validation data and the remaining is used for training. The cross-validation process is then repeated $k$ times (the folds). The average of $k$ results from the folds gives the test accuracy of the algorithm [21].

In our study, we used 5-fold cross validation. The average of these five fold gave the test result for each fold. 


\section{RESULTS}

In this study, we found several networks with same best accuracy. The best net consisted of total 6 inputs according to 6 color parameters, where 3 parameters came from crossed-polarized images and 3 came from plane-polarized images as seen in Figure 1. The networks were trained with each having a different number of hidden nodes. 5-fold-cross validation method was involved to select data and MLPNN with one hidden layer was used. The classification of mineral using ANN proved \%93.86 accuracy for 400x6 data with 6x21x5 MLPNN structure trained in 8000 epoch.

In second study, multiple neural networks were implemented for each of the quartz, muscovite, biotite, chlorite and opaque as shown in Figure 5. Size of data set was same with previous data. Each network model was differed from each other. Also 5-fold-cross validation method was involved to select data and MLPNN with one hidden layer was used. While the inputs of five different networks are the same, their outputs are differing from each other [22]. Table 1 presents the best network architecture and shows a complete listing of the types and percentage of classification accuracy of minerals which were identified. For each mineral, the average accuracy of the network is greater then $90 \%$.

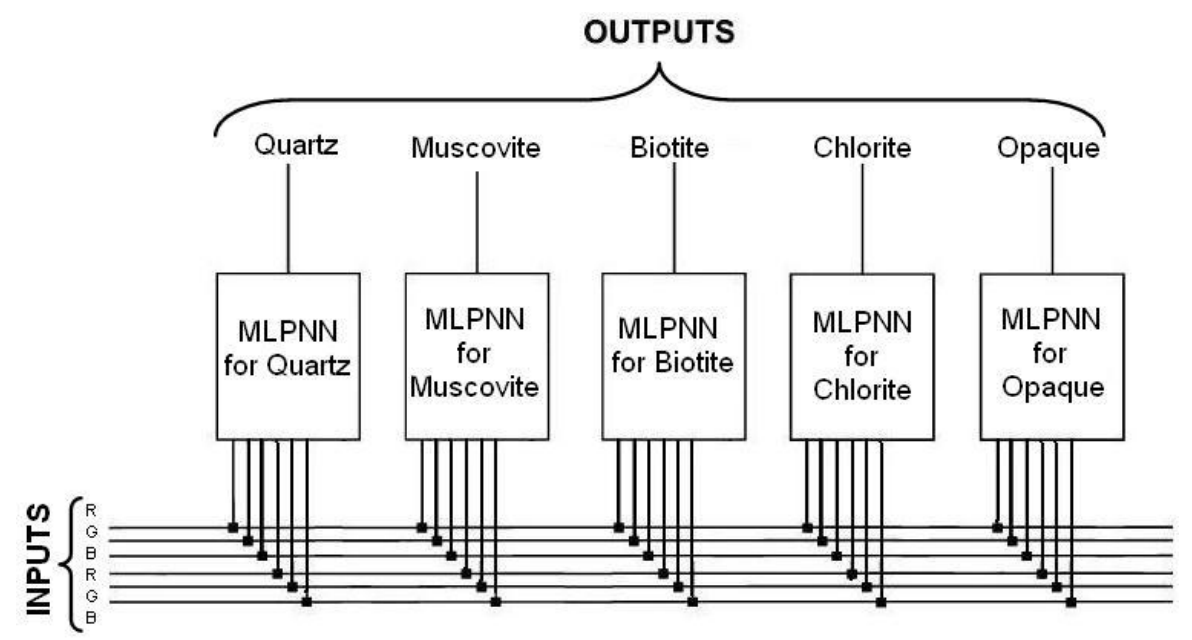

Figure 5. Prepared combined networks for mineral classification

Table $1-$ ANN results

\begin{tabular}{|l|c|c|c|c|}
\hline & MLPNN structure & Number of epoch & Training accuracy & Test accuracy \\
\hline Quartz & 6x196x1 & 8000 & 98.40 & 90.67 \\
\hline Muscovite & 6x13x1 & 7000 & 99.69 & 96.16 \\
\hline Biotite & 6x66x1 & 9000 & 98.18 & 93.91 \\
\hline Chlorite & $6 \times 86 \times 1$ & 9000 & 98.86 & 92 \\
\hline Opaque & 6x21x1 & 3000 & 98.34 & 97.62 \\
\hline
\end{tabular}




\section{CONCLUSION}

According to study, the results show that a well designed and trained with a high quality large size data set ANN can be used for classification of mineral. ANN can distinguish and classify the minerals according to their colors.

In this study, we used ANN to classify the minerals with more than $90 \%$ accuracy. It can be improved with a larger data set or other parameters like texture. Different ANNs can be used in practical applications for mineral classification.

\section{ACKNOWLEDGEMENTS}

The author would like to thank Gürsel Kansun for manually identifying the minerals used in this study. The authors are grateful to Selçuk University Scientific Research Projects Coordinatorship (Project:08101002) and TUBITAK (The Scientific and Technological Research Council of Turkey) for after press support of the manuscript.

\section{REFERENCES}

1. G.A.Hope; R. Woods; C.G. Munce, Raman microprobe mineral identification. Minerals Engineering 14(12), 1565-1577, 2001

2. N.A. Baykan and N. Y1lmaz, Mineral identification using color spaces and artificial neural networks. Computers \& Geosciences 36(1), 91-97, 2010

3. M. E. Petersen; D. Ridder; H. Handels, Image Processing with neural network-a review. Pattern Recognition 35, 2279-2301, 2002

4. E. Kaczmarek; T.M. Brzozowska; B. Miskowiak, Digital image analysis in dental research applied for treatment of fissures on occlusal surfaces of premolars. Annals of Biomedical Engineering 31, 931-936, 2003

5. S. Li; T. Fevens; A. Krzyzak; S. Li, Automatic clinical image segmentation using pathological modeling, PCA and SVM. Engineering Applications of Artificial Intelligence 19, 403-410, 2006

6. P.J. Sjöström; B.R. Frydel.; L.U. Wahlberg, Artificial neural network-aided image analysis system for cell counting. Cytometry 36, 18-26, 1999

7. D.T. Pham and S. Sagiroglu, Training multilayered perceptrons for pattern recognition: A comparative study for four training algorithms. International Journal of Machine Tools and Manufacture 41(3), 419-430, 2001

8. İ. Güler and E.D. Übeyli, ECG beat classifier by combined neural network model. Pattern Recognition 38(2), 199-208, 2005

9. A.H. Bagherieh; J.C. Hower; A.R. Bagherieh; E. Jorjani, Studies of the relationship between petrography and grindability for Kentucky coals using artificial neural network. International Journal of Coal Geology 73(2), 130-138, 2008

10. İ. Güler and E.D. Übeyli, Detection of ophthalmic arterial doppler signals with Behcet disease using multilayer perceptron neural network. Computers in Biology and Medicine 35(2), 121-132, 2005

11. F. Fueten, A computer-controlled rotating polarizer stage for the petrographic microscope. Computers \& Geosciences 23(2), 203-208, 1997 
12. R.C. Gonzales and R.E. Woods, Digital Image Processing. Addison-Wesley Publishing Company, USA, 1993

13. R.L. Swenson and K.R. Dimond, A universal colour transformation architecture. Pattern Recognition Letters 19(9), 805-813, 1998

14. S. Sagiroglu and N. Yilmaz, Web-based mobile robot platform for real-time exercises. Expert Systems with Applications 36(2), 3153-3166, 2009

15. I.A. Basheer and M. Hajmeer, Artificial neural networks: fundamentals, computing, design, and application. Journal of Microbiological Methods 43(1), 3-31, 2000

16. B.B. Chaudhuri and U. Bhattacharya, Efficient training and improved performance of multilayer perceptron in pattern classification. Neurocomputing 34, 11-27, 2000

17. E.D. Übeyli and İ. Güler, Multilayer perceptron neural networks to compute quasistatic parameters of asymmetric coplanar waveguides. Neurcomputing 62 , 349-365, 2004

18. E. Öztemel, 2003. Yapay Sinir A ğları. Papatya Publisher, Turkey, 2003

19. S. Serhatlığlu; F. Hardalaç; İ. Güler, Classification of transcranial Doppler signals using artificial neural network, Journal of Medical Systems 27(2), 205-214, 2003

20. D. François; F. Rossi; V. Wertz; M. Verleysen, Resampling methods for parameterfree and robust feature selection with mutual information. Neurocomputing 70 (79), 1276-1288, 2007

21. N.A. Diamantidis; D. Karlis; E.A. Giakoumakis, Unsupervised stratification of cross-validation for accuracy estimation. Artificial Intelligence 116 (1-2), 1-16, 2000

22. I. Babaoglu; O.K. Baykan; N. Aygul; K. Ozdemir; M. Bayrak, Assesssment of exercise stress testing with artificial neural network in determining coronary artery disease and predicting lesion localization. Expert Systems with Applications 36, 2562-2566, 2009 